\title{
Open Access publizieren in der „wiener klinischen wochenschrift"
}

Alois Sillaber

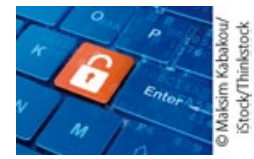

Online publiziert: 26. Januar 2016

(C) Springer-Verlag Wien 2016

Wissenschaftler in Österreich können ab 2016 in über 1600 Springer-Zeitschriften - darunter auch der „wiener klinischen wochenschrift"Open Access publizieren

Springer und das Bibliothekskonsortium in Österreich (Kooperation E-Medien Österreich/KEMÖ) haben im Herbst 2015 ein weitreichendes, neues Lizenzmodell „Springer Compact“ mit dreijähriger Laufzeit ab Januar 2016 vereinbart. Springer Compact verbindet die Nutzung von Inhalten auf SpringerLink mit der Möglichkeit, Open Access-zu publizieren. Die Konsortialmitglieder erhalten Zugriff auf über 2000 Springer-Zeitschriften und können in über 1600 Springer Hybrid-Zeitschriften (wie z. B. der „wiener klinischen wochenschrift“) Open Access publizieren.

Springer hat ein vergleichbares Modell mit den niederländischen Universitäten, der Max Planck Gesellschaft und in Großbritannien vereinbart. Das neue Modell bietet WissenschaftlerInnen hervorragende Möglich- keiten, ihre Forschungsergebnisse in einem qualitativ hochwertigen und breiten Zeitschriftenportfolio Open Access zu publizieren. Sie können sich auf das Publizieren konzentrieren und müssen sich nicht um die finanziellen Rahmenbedingungen und administrativen Anforderungen im Hinblick auf OA-Mandate kümmern.

Gültig ist diese Regelung für alle Manuskripte, die nach dem 1.1.2016 akzeptiert werden, d. h. es können auch Artikel, die schon vor 2016 eingereicht wurden, berücksichtigt werden.

Bei Fragen zu den Möglichkeiten der OA-Publikation in der „wiener klinischen wochenschrift" wenden Sie sich bitte an: oa.verification@springer.com. Weitere Informationen finden Sie unter http://www.springer. com/de/open-access/springer-open-choice/agreements/750588

Dr. Alois Sillaber

Managing Director

Springer Wien 